\title{
Self-apposing stent fracture in the context of myocardial bridging leading to in-stent chronic total occlusion: When the muscle trumps the metal
}

\author{
Lorenzo Azzalini, Marco B. Ancona, Satoru Mitomo, Mauro Carlino, Antonio Colombo \\ Interventional Cardiology Unit, Cardio-Thoracic-Vascular Department, \\ San Raffaele Scientific Institute, Milan, Italy
}

A 55-year-old man presented with angina and severe anteroapical ischemia. He had suffered non-ST-elevation myocardial infarction 15 months earlier at another institution. The culprit lesion was located on the mid left anterior descending (LAD), in the context of myocardial bridging (MB) with systolic compression (milking effect) (Fig. 1A). Three overlapping drug-eluting stents (DES), including two self-apposing Stentys Xposition S, were implanted in the MB segment with optimal result (Fig. 1B).

Coronary angiography revealed in-stent chronic total occlusion of the mid LAD, associated with fracture of the most proximal Xposition S (Fig. 1C). The lesion was crossed antegradely and predilated. Optical coherence tomography confirmed self-apposing stent fracture (Fig. 1e-g). After further lesion preparation with non-compliant and scoring balloons, a $3.0 \times 28 \mathrm{~mm}$ Boston Scientific Synergy DES was implanted and post-dilated. The distal vessel was treated with a $2.5 \times 30 \mathrm{~mm}$ drugeluting balloon. Optimal angiographic (Fig. 1D) and optical coherence tomography (Fig. 1e'-g') result was observed. The patient was asymptomatic at 9-month follow-up, and a stress-echocardiogram was negative for ischemia.
Stent fracture is a rare cause of occlusive instent restenosis. It may result from excessive mechanical stress on the vessel wall that occurs from repetitive contraction and/or flexion of the vessel, as it is seen in stented segments within a MB with milking effect. Patients may be asymptomatic, or present with an acute coronary syndrome, which is suggestive of stent thrombosis, or recurrent angina, thus suggesting in-stent restenosis, as in the presented case.

It was speculated that the rationale behind implanting self-apposing stents in this patient was the fact that they exert a continuous, gentle outward force onto the vessel wall, which would have counteracted systolic compression during repetitive cardiac cycles. However, the integrity of this kind of stent in the context of MB with milking proved to be insufficient. To treat occlusive in-stent restenosis, we chose the thin-strut, platinum-chromium, bioabsorbable-polymer, everolimus-eluting Synergy stent, due to its high radial force. This report suggests thorough reflection before considering stent implantation in a $\mathrm{MB}$, since this approach can lead to stent fracture with subsequent (occlusive) in-stent restenosis, which poses challenges with regards to subsequent patient management.

Conflict of interest: None declared

\footnotetext{
Address for correspondence: Lorenzo Azzalini, MD, PhD, MSc, Interventional Cardiology Division, Cardio-Thoracic-Vascular Department, San Raffaele Scientific Institute, Via Olgettina 60, 20132 Milan, Italy, tel: +39 0226437331 , fax: +3902 2643 7339, e-mail: azzalini.lorenzo@hsr.it
} 


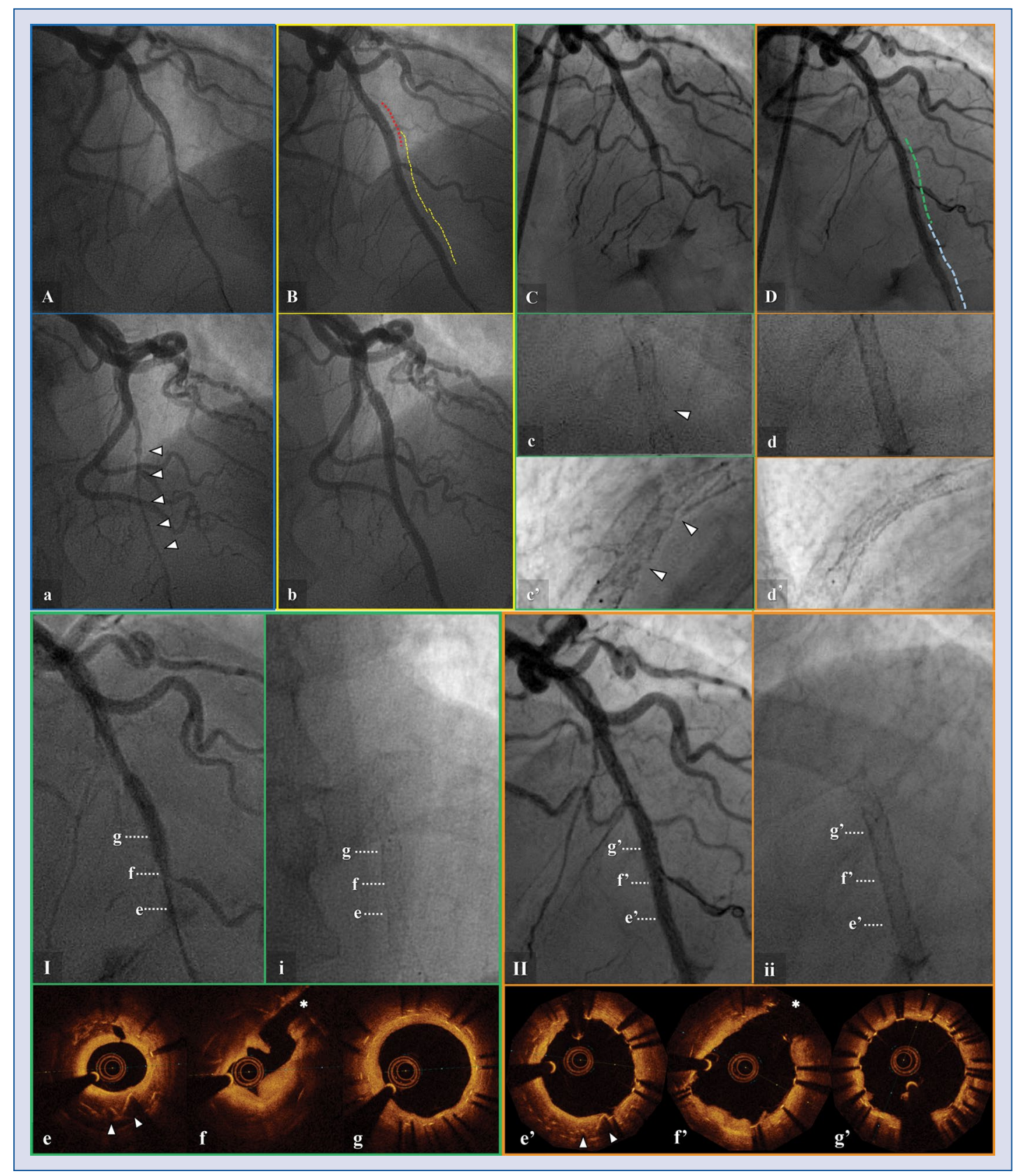

Figure 1. Baseline coronary angiography showing myocardial bridging with milking (A. Diastole, a. Systole; arrowheads: milking). Percutaneous coronary intervention was performed (red line: Biomatrix $3.5 \times 11$ mm; yellow lines: Stentys 3.0-3.5 $\times 27 \mathrm{~mm}$ and 2.5-3.0 $\times 22 \mathrm{~mm}$; B. Diastole, b. Systole). Fifteen months later, chronic total occlusion was documented (C), with plain fluoroscopy showing fractured stent at two sites (arrowheads; c. Cranial view, $\mathbf{c}^{\prime}$. Lateral view). Additional drug-eluting stent (DES) implantation, as well as treatment with drug-eluting balloon (DEB), were performed (D, green and light blue line, respectively), with good result (d. Cranial view, d'. Lateral view). I. Coronary angiography and (i) plain fluoroscopy after in-stent occlusion recanalization with a small balloon. e-g. Optical coherence tomography (OCT) images at each level indicated in I and i. e. Arrowheads indicate fractured stent struts, f. Asterisk indicates side branch, g. Overlap of previous stents. Final result (II. Coronary angiography, ii. Plain fluoroscopy) after revascularization with DES and DEB. $\mathbf{e}^{\prime}-\mathbf{g}^{\prime}$. OCT images at each level indicated in II and ii. e'. Arrowheads indicate fractured stent struts compressed by new DES. f'. Asterisk indicates side branch. $\mathbf{g}^{\prime}$. Multiple overlapping struts of previous and new stents. 